\title{
STRAFRECHTSDUALISMUS UND STRAFGERICHTSBARKEIT IN NIGERIA
}

\author{
Von Barbara Huber
}

\section{Vorbemerkung}

Im heutigen Nigeria gelten - mit unbedeutenden Abänderungen in den einzelnen Bundesstaaten - zwei Strafgesetzbücher: der Nigerian Criminal Code von 1916 (NCC) in Lagos und in den südlichen Staaten ${ }^{1}$ und der Nigerian Penal Code von 1959 (NPC) in den Nordstaaten ${ }^{2}$. Entsprechend unterschiedlich sind das Prozeßrecht und der Gerichtsaufbau geregelt. Ziel dieses Aufsatzes soll es sein, die historischen Hintergründe, die zu dem modernen Rechtsdualismus in einem afrikanischen Staat geführt haben, nachzuzeichnen und damit einen vorläufigen Beitrag zur strafrechtlichen Grundlagenforschung im nigerianischen Raum zu bieten. Voraussetzung für das Verständnis des nigerianischen Strafrechtsdualismus ist eine gewisse Kenntnis der ethnischen Zusammensetzung dieses Mehrvölkerstaates sowie der verwaltungs- bzw. verfassungsmäßigen Struktur seit der Errichtung der britischen Herrschaft in Westafrika.

\section{I. Überblick über die Stammeseinteilung und verfassungsgeschichtliche Entwicklung}

1. Die Sozialstruktur des nigerianischen Staates wird, wie wohl aller Staaten Schwarzafrikas, durch die Stämme bestimmt. Im Gegensatz zu modernen europäischen Staaten, in denen der einzelne Bürger als Individuum der Mittelpunkt sozialer Beziehungen ist, bilden in Afrika noch immer der Stamm und die ihn formenden Einheiten wie Clan und Familie das Zentrum des Soziallebens, dem die Loyalität jedes Angehörigen dieser Einheit gehört.

Grob gesprochen läßt sich Nigeria in drei große Stammesgebiete einteilen: die Yoruba leben überwiegend im Westen, die Ibo im Osten und die Haussa/Fulani im Norden. Yoruba, Ibo und Haussa gehören zur negroiden Rasse, die Fulani jedoch sind Hamiten, d. h. sie sind keine Neger und heben sich durch hellere Haut von den übrigen Bewohnern des Landes $\mathrm{ab}^{3}$.

Kennzeichnender Zug der politischen wie der rechtlichen Geschichte ist jedoch nicht diese Einteilung $\mathrm{zwischen}$ negroid und hamitisch, sondern die Tatsache, daß sowohl die Haussa wie die Fulani den Islam als Staatsreligion betrachteten. Auf

\footnotetext{
1 Western Nigeria, Midwestern State; Eastern Nigeria, East-Central State, River State und South-Eastern State.

2 Kwara, Northwest-State, North-Central State, Kano, Benue-Plateau State, North-East State.

Diese Arbeit wird fortgesetzt durch ein von der Deutschen Forschungsgemeinschaft finanziertes Forschungsprojekt des Max-Planck-Instituts für ausländisches und internationales Strafrecht, Freiburg i. Br. über die Behandlung der Tötungsdelikte in Nigeria.

3 Die Herkunft der Fulani, die auch Peul, Ful, Fulbe, Fulbata und Felattiya genannt werden, ist umstritten. Siehe dazu: Meyer's Handbuch über Afrika, Mannheim 1962, S. $185 \mathrm{f}$.

Die Fulani unterwarfen 1804 die Haussa und errichteten ein gut funktionierendes hierarchisch geordnetes Feudalreich, in dem sie sich als Herrenschicht etablierten. Sie paßten sich jedoch mit der Zeit der Lebensweise der einheimischen Haussa an, mit denen sie von Anbeginn die gemeinsame Religion, der Islam, verband.
} 
diesem kuturellen Nährboden hat sich im Norden ein eigenständiges Volkstum ${ }^{4}$ mit einem großen Grad kultureller Einheitlichkeit dank einheitlicher Sprache, Kultur, Sitte und Religion entwickelt. Die Unterschiede zwischen den mohammedanischen Nordbewohnern und den Südstämmen liegen somit nicht an stammesmäßigen Andersartigkeiten, sondern es handelt sich um verschiedene Völker, die sich in Kultur und Religion voneinander abheben ${ }^{5}$. Der mohammedanische Norden ist eher konservativ, Veränderungen abgeneigt, andersartigen Ideen gegenüber feindlich eingestellt und selbstbewußt; der Süden ist demgegenüber fortschrittlich, aufgeschlossen auch gegenüber anderen Religionen ${ }^{6}$ und hat durch den ständigen wirtschaftlichen Kontakt mit europäischen Handelspartnern, durch die Übernahme moderner Wirtschaftsmethoden ${ }^{7}$ sowie durch den den Import und Export fördernden Zugang zum Meer eine spürbare wirtschaftliche Überlegenheit gegenüber dem Norden erlangt, die zur Vertiefung des Grabens zwischen Nord und Süd in diesem Lande beigetragen hat ${ }^{8}$. Die tiefen kulturellen Unterschiede konnten auch dadurch nicht überbrückt werden, daß beide Teile des großen Gebietes ein halbes Jahrhundert lang unter einer gemeinsamen Regierung mit weithin einheitlichem Verwaltungs-, Wirtschafts- und Rechtssystem gelebt haben.

2. Die politische Vereinigung zwischen Norden und Süden fand 1914 statt, als die Kolonie Lagos, die Protektorate Südnigeria ${ }^{9}$ und Nordnigeria ${ }^{10}$ zur Kolonie und Protektorat Nigeria unter einem Generalgouverneur zusammengefaßt wurden.

Dies bedeutete für die Justizverwaltung eine Vereinfachung, indem es von diesem Zeitpunkt an nur noch ein Oberstes Gericht für das ganze Gebiet gab. Entsprechend gab es nur noch einen Chief Justice und einen Attorney General. Nicht vereinheitlicht wurde aber die Gesetzgebung. Von der Proklamierung der Protektorate im Jahre 1900 an war bis 1906 in Südnigeria und bis 1914 in Nordnigeria

4 Westermann: Die Volkswerdung der Haussa. Berlin 1950, S. 19.

5 Die durch den Islam genährte fatalistische Lebenseinstellung bedingt ein völlig anderes Verhältnis des einzelnen $\mathrm{zu}$ individueller Leistung, materiellem Reichtum oder politischem Einfluß, zu geschäftlichem Unternehmergeist, als sie z. B. bei den Ibos zu finden ist. Die stark individuell und elitär bestimmte Sozialstruktur dieses Stammes, in dem soziale und politische Macht auf einzelne Familien verteilt ist, hat zu der Ưberzeugung geführt, daß individuelle Selbstbestimmung, materieller Wohlstand und demokratische Gleichheit erstrebenswerte Errungenschaften sind.

6 Das Christentum wurde insbesondere von den Ibos angenommen und hat großen Einfluß auf die Erziehung und Bildung der südlichen Bevölkerung gehabt.

7 Abwendung von der Subsistenzwirtschaft zur Ertragswirtschaft durch die Anpflanzung von Kakao, Kaffee, Gummibäumen und Palmen.

8 Die Gegensätze zwischen beiden Regionen führten zu Aufständen wie dem Kano-Aufstand 1953 und letztlich auch zum Biafra-Krieg. 1949 beschrieb Mallam Abubakar, ein führender Politiker aus dem Norden, das Verhältnis zum südlichen Nachbarn mit den Worten: We despise each other, we call each other ignorant; the South is proud of Western knowledge and culture; we are proud of Eastern (culture). To tell you the truth, the common people of the North put more confidence in the white man than in their Southern brother, zitiert nach: Nwabueze: Constitutionalism in the Emergent States, London 1973, S. 88 .

9 Nach Abschluß des Zessionsvertrages im Jahre 1861, durch den das Gebiet der Stadt Lagos von den afrikanischen Häuptlingen an die britische Krone abgetreten wurde, dehnte sich der Einflußbereich der Handelskompanien bis weit in das Hinterland hinein aus. Die Stammesführer schlossen Zessionsverträge, durch die die Gebietshoheit auf die Handelskompanien überging „o cede the whole of our territory to the Company, their heirs and assigns for ever“ ... vgl. Nwabueze: Constitutional Law of the Nigerian Republic, London 1964, S. 9 ff. Dies führte 1900 zur Proklamation dieses Gebietes als Protektorat und 1906 zur verwaltungsmäßigen Vereinigung des Protektorates Südnigeria mit der Kolonie Lagos, deren Gebiet sich infolge weiterer Abtretungen an die britische Krone bis zu diesem Zeitpunkt weit ins Yoruba-Land hinein ausgedehnt hatte. Sitz der gemeinsamen Verwaltung war Lagos. Das Gebiet wurde in Provinzen aufgeteilt - Western, Eastern, Central Province, mit je einem Provincial Governor an der Spitze. Diese Provincial Governors wiederum unterstanden dem Gouverneur von Lagos. $\mathrm{Zu}$ weiteren Einzelheiten siehe Elias: Nigeria: Development of its Laws and Constitutiono, London, 1967, S. $15 \mathrm{ff}$.; Nwabueze: Constitutional Law of the Nigerian Republic, S. $9 \mathrm{ff}$.

10 Das Gebiet nördlich des Niger wurde ebenfalls 1900 zum Protektorat erklärt. Erster High Commissioner des Gebietes wurde Sir F. Lugard, der hier sein System der "indirect rule entwickelte, indem er das wohlausgebaute Verwaltungssystem der Emirate übernahm und" das Gebiet weitgehend in der Hand der eingeborenen Ordnungsorgane beließ. - Lugard: The Dual Mandate in British Tropical Africa, Edinburgh, 1922. 
allein der englische Gouverneur dieser Gebiete gesetzgebendes Organ ${ }^{11}$. Die von ihm vorgeschlagenen Gesetze bedurften der Zustimmung des englischen Königs, um geltendes Recht zu werden. Die Gesetze wurden in Form von Ordinances erlassen.

Zuständig für den Erlaß von Gesetzen im Bereich der Kolonie Lagos war seit 1862 der Legislative Council for the Colony ${ }^{12}$, der von 1906 bis zum Zusammenschluß der Gebiete Nordnigeria, Südnigeria und der Kolonie Lagos 1914 seine Zuständigkeit auf den gesamten südnigerianischen Raum ausdehnte ${ }^{13}$. Nach 1914 wurde seine Zuständigkeit wiederum auf den Bereich der Kolonie Lagos beschränkt ${ }^{14}$, während der Gouverneur die gesetzgebende Autorität für die zusammengeschlossenen Protektorate wurde ${ }^{15}$. Erst mit der Verfassung von 1922 wurde ein Legislative Council of Nigeria eingerichtet, der jedoch entgegen seiner Bezeichnung nicht für den gesamten nigerianischen Raum, sondern nur für die Kolonie Lagos und die südlichen Provinzen zuständig war ${ }^{16}$. Gesetzgebende Gewalt für die nördlichen Provinzen blieb allein der Gouverneur ${ }^{17}$.

Auch in dieser Phase der nigerianischen Entwicklung bot sich keine Gelegenheit, die starken kulturellen Gegensätze zwischen den nördlichen und südlichen Völkern zu mildern ${ }^{18}$. Die gesetzgeberische Zentralisierung wurde erst durch die Verfassung von 1946 erzielt, als der Nigerian Legislative Council errichtet wurde, der Einfluß auf die Gesetzgebung des Gouverneurs ausüben konnte, da nunmehr die Zusammensetzung des Council ihm eine tatsächliche Macht einräumte ${ }^{19}$. Hier bot sich zum ersten Mal eine zentrale Plattform für den dringend gebotenen Dialog zwischen Nord und Süd.

Die verfassungsmäßige Situation war jedoch dank der Selbständigkeitsbestrebungen der nördlichen Regionen nicht von Dauer. Diese Gebiete bedauerten den $\mathrm{Zu}$ sammenschluß von 1914 und verlangten die Wiederherstellung einer eigenständigen Nordregierung mit voller Autonomie in internen Angelegenheiten. Unter dem Eindruck der Unruhen in Kano 1953 wurde daher mit der Verfassung von 1954 Eine Föderation geschaffen, die den tief eingewurzelten Unterschiedlichkeiten so-

$11 \mathrm{Er}$ war befugt: „by proclamation to provide for the administration of justice, the raising of revenue, and generally for the peace, order and good of the country and all persons therein, including the prohibition and punishment of each tending to disturb the public peace." - Northern Nigeria Protectorate Order in Council, 1899, Art. 6; Southern Nigeria Protectorate Order in Council, 1899, Art. 6.

12 Der Legislative Council war ein beratendes Gremium, in dem neben weißen Einwohnern auch vereinzelte Vertreter der schwarzen Bevölkerung saßen. Die Errichtung dieses Organs bedeutete formal eine Beschränkung der Gesetzgebungsgewalt des Gouverneurs, denn er konnte nur noch mit der Zustimmung und nach Beratung mit dem Council Gesetze erlassen. Da diese Zustimmung jedoch immer gegeben wurde, also lediglich eine förmliche Angelegenheit war - die Councils bis 1922 waren überwiegend mit Angestellten des öffentlichen Dienstes, die vom Gouverneur ernannt wurden, besetzt - bildete der Council lediglich ein Instrument, um den britischen Beamten ein Bild der Meinung der Bevölkerung zu verschaffen; der Council war kein. Organ der Mitbestimmung. Siehe im einzelnen zur Zusammensetzung und Aufgabe: Nwabueze, Constitutional Law of the Nigerian Republic, S. $48 \mathrm{ff}$.; insbesondere S. 54.

13 Southern Nigeria Protectorate Order in Council, 1906, Art. 4.

14 Letters Patent vom 29. November 1913, Art. 7. Die Beschränkung wurde vom ersten Gouverneur, Sir Lugard, mit der schlechten Infrastruktur des Landes begründet, die es nigerianischen Vertretern im Council unmöglich machte, regelmäßig im gesamtnigerianischen Gremium mitzuarbeiten. - Siehe Extraordinary Gazette, No. 17, 1. Januar 1914, S. 18 - zitiert nach Nwabueze, Constitutional Law of the Nigerian Republic, S. 46, Anm. 3.

15 Nigeria Protectorate Order in Council, 1913, Art. 8.

16 Nigeria Protectorate Order in Council, 1922, Art. 9; Nigeria (Legislative Council) Order in Council, 1922, Art. 23. Der Legislative Council for the Colony wurde abgeschafft.

17 Protectorate Order, 1922, Art. X. Wegen weiterer Einzelheiten siehe Nwabueze: Constitutional Law of the Nigerian Republic, S. 44 ff.

18 Coleman: Nigeria: Background to Nationalism, Berkeley, 1963, S. 360.

$19 \mathrm{Die}$ sog. Richard-Verfassung trat am 1. Januar 1947 in Kraft. Nigeria (Legislative Council) Order in Council, 1946, S. 4. Der Rat war jetzt zusammengesetzt aus dem Gouverneur als Präsidenten, 16 Beamten (13 kraft Amtes und drei vom Gouverneur Benannten) sowie 28 Nicht-Beamten (24 ernannt und 4 gewählt). Die gewählten Nicht-Beamten wurden von den Regional Councils, beratenden quasi parlamentarischen Gremien in den Provinzen entsandt. Ihre Aufgabe war die Vermittlung zwischen dem zentralen Organ, dem Legislative Council und den Native Authorities. Elias, Development, S. $35 \mathrm{f}$. 
wohl zwischen Nord und Süd wie zwischen den gößeren südlichen Stämmen, Ibo und Yoruba, Rechnung trug ${ }^{20}$. Die Justiz wurde regionalisiert: Anstelle des einen bestehenden Supreme Court mit seinen zehn Abteilungen für das ganze Land wurde in jeder Region und in Lagos ein High Court errichtet, gegen dessen Entscheidungen Rechtsmittel zum Federal Supreme Court in Lagos gegeben waren. Auch der öffentliche Dienst gelangte in die Zuständigkeit der einzelnen Regionen, was insbesondere im Norden $\mathrm{zu}$ einer extremen Regionalisierung führte ${ }^{21}$. Die föderative Konstruktion begünstigte außerdem in den Regionen eine Haltung der Selbstgenügsamkeit, des Separatismus und der Intoleranz anderen Gebieten gegenüber. Hinzu trat das Ungleichgewicht zwischen der Größe und der Bevölkerungszahl des Nordens gegenüber den beiden südlichen Regionen ${ }^{22}$ sowie die wirtschaftliche und bildungsmäßige Überlegenheit der südlichen Teile. Das föderative System wurde auch nach der Erlangung der Selbständigkeit ${ }^{23}$ beibehalten, und die Aufteilung des Landes im immer mehr selbständige Bundesländer (regions) schritt trotz der unitarischen Phase der Militärregierung nach $1966^{24}$ fort ${ }^{25}$ und endete 1967 in einer Zwölfstaatenstruktur der Militärregierung ${ }^{26}$.

\section{Quellen des nigerianischen Strafrechts}

Die beiden geltenden Codes, der Criminal Code und der Penal Code sind zu stark voneinander abweichenden Zeitpunkten, nämlich 1916 und 1959 eingeführt worden; sie haben beide als Großmutterrecht das englische Common Law. Interessant sind der Weg, den dieses englische Recht genommen hat, und die Gründe, die zur Einführung von zwei verschiedenen Gesetzbüchern geführt haben.

1. Nach mehreren vergeblichen Versuchen, in der Kolonie Lagos ein Strafgesetzbuch einzuführen ${ }^{27}$, ergriff schließlich im Jahre 1903 der Chief Justice des nördlichen Protektorats die Initiative und entwarf einen Code, als dessen Vorlage er nach gründlichen Studien der seinerzeit geltenden oder entworfenen Codes ${ }^{28}$ den

20 Nigeria wurde jetzt ein Bundesstaat, bestehend aus den drei Regionen: Nordnigeria, Westnigeria, Ostnigeria sowie dem Bundesgebiet Lagos als Hauptstadt. Die Verfassung folgte dem australischen Beispiel. Elias, Development S. $41 \mathrm{ff}$.

21 Hier wurde das Motto ausgegeben: One North, one People. Es begann eine Northernization, aufgrund derer bei der Besetzung öffentlicher Stellen einem Angehörigen der Nordregion gegenüber Südnigerianern der Vorzug gegeben wurde; Südnigerianer durften in Nordnigeria keinen Grund und Boden mehr erwerben und schließlich wurde sogar die Pachtung von Land durch nicht zu Nordnigeria gehörende Stammesangehörige ohne Zustimmung der Regierung als strafbare Handlung angesehen. Nwabueze: Constitutionalism in Emergent States, S. 130.

22 Der Norden umfaßte $75 \%$ des Staatsgebietes und $60 \%$ der Gesamtbevölkerung.

23 Nigeria (Constitution) Order in Council 1960; Nigeria Independance Act 1960.

24 Constitution (Suspension and Modification) Nr. 5 Decree of 1966.

$25 \mathrm{Zu}$ den Regionen Nord-, West-, Ostnigeria sowie Lagos kam 1964 der Mid Western State hinzu. Das Gebiet umfaßt Benin und die Deltaregion; es wird von Nichtyorubas bewohnt und wurde deshalb von Westnigeria, dem traditionellen Yorubagebiet, abgetrennt. Siehe "The creation of the mid west region". In: 1 Nigeria Law Journal, 1964, S. $100 \mathrm{ff}$.

26 States Creation Decree 1967: Die Nordregion wurde aufgeteilt in sechs Staaten: Kano, Kwara, NorthEastern-State, North-Western-State, North-Central-State und Benue-Plateau-State. Im Süden wurden der Western State, Mid-Western State und Lagos beibehalten; der Eastern State wurde aufgeteilt in die South Eastern, East Central und River States.

27 Im Jahre 1889 forderte das Kolonialministerium die Gouverneure von Sierra Leone, der Goldküste, Gambias und Lagos auf, nach dem Modell des Code von St. Lucia einen Strafgesetzentwurf vorzulegen; dieser. Aufforderung kam jedoch nur die Goldküste nach. Erst neun Jahre später wandte sich der damalige Gouverneur von Lagos, McCallum, an das Kolonialministerium mit der Bitte nach einem Code. Ein auf dem Gesetzbuch der Goldküste beruhender Entwurf wurde 1899 dem Legislative Council vorgelegt, jedoch aus politischen Gründen nicht angenommen. Siehe dazu ausführlich: Morris, How Nigeria got its Criminal Code. 1970 J. A. L., S. $137 \mathrm{ff}$.

28 Criminal Code der Gold Coast Colonie von 1892, beruhend auf dem Code von St. Lucia; Sir James R. Stephen's Entwurf eines Strafgesetzbuchs für England; Sudan Penal Code von 1899 und Indian Penal Code von 1860. 
Queensland Criminal Code von 1899 benutzte, weil dieser Code seiner Meinung nach die beiden Mängel vermied, die rezipierte Gesetzbücher so oft aufwiesen: zu genaue Ausarbeitung auf der einen Seite und zu stark vereinfachende Zusammenfassung auf der anderen Seite ${ }^{29}$. Das von ihm entworfene Strafgesetzbuch wurde in Nordnigeria im Jahre 1904 eingeführt.

In Unkenntnis der Einführung eines Strafgesetzbuches in Nordnigeria fragte der Gouverneur von Südnigeria 1904 erneut im Kolonialministerium an, ob es nicht an der Zeit sei, in Südnigeria einen Strafcode einzuführen und schlug vor, den Straits Settlements Code als Grundlage eines Entwurfs zu benutzen ${ }^{30}$. Seiner Ansicht nach sei dieser in den Straits Settlements bewährte Code wegen seiner Verständlichkeit und Vollständigkeit besonders gut für eine ähnlich strukturierte Bevölkerung, wie sie in Lagos und in Südnigeria zu finden sei, geeignet. Seine Argumente fanden im Kolonialministerium in London kein Gehör ${ }^{31}$, das im Gegensatz zu den Vorschlägen des Gouverneurs die Einführung des in Nordnigeria bereits geltenden Code auch in Lagos und Südnigeria befürwortete, ohne daß hierüber eine endgültige Entscheidung getroffen wurde ${ }^{32}$. Südnigeria und Lagos blieben also bis zur Vereinigung mit dem nordnigerianischen Protektorat im Jahre 1914 ohne ein Strafgesetzbuch; es galten das customary law, die in Lagos und im Protektorat selbst erlassenen Gesetze (ordinances and proclamations) sowie common law and statutes des Vereinigten Königreichs, soweit ihre Geltung auf die Kolonien erstreckt wurde.

Erst nach der Zusammenfassung der Protektorate Nord- und Südnigeria sowie der Kolonie Lagos im Jahre 1914 unter dem Gouverneur Lugard wurde der Northern Criminal Code mit einigen Abänderungen 1916 im Süden eingeführt, wo er eine Zeitlang starke Oppostion auslöste. Von diesem Zeitpunkt an galt bis 1960 in ganz Nigeria ein einheitliches Strafgesetzbuch, das seinerseits wiederum das Vorbild für einen Musterentwurf des Kolonialministeriums in London bildete und in den zwanziger Jahren in Ostafrika, Nyassaland (heute Malawi), Nordrhodesien (heute Sambia) und Gambia eingeführt wurde.

Die Einführung und Geltung eines einheitlichen Strafgesetzbuches, die weniger das Ergebnis einer sorgsamen Planung und einheitlichen Kriminalpolitik des Kolonialministeriums, sondern eher die Folge von einzelnen Zufällen war ${ }^{33}$, hatte nicht zur Folge, daß sich in Nigeria in der Zeit von 1916 bis 1960 auch ein einheitliches Strafrecht herausbildete. Die kulturellen Gegensätze waren stärker als das einheitliche Gesetzbuch; sie führten schließlich wieder zu abweichenden Codes. Bevor jedoch hier auf die jüngsten Ereignisse eingegangen wird, möchte ich die Rechtslage schildern, wie sie sich vor und nach der Einführung des Code in den beiden Teilen des Landes bot.

29 Am Code der Gold Coast Colonie bemängelte er insbesondere, daß die die Tatbestandsmerkmale definierenden Bestimmungen weggelassen worden waren, was den meist nicht juristisch vorgebildeten Richtern die Arbeit mit dem Gesetz und das Verständnis der einzelnen Vorschriften sowie der gesetzlichen Zusammenhänge erschwerte. Vgl. Report on (draft) Criminal Code Proclamation, Dispatch vom 6. 10. 1903 C. O. 446/33 - abgedruckt bei Morris, a. a. O., S. 144.

30 Der Straits Settlements Code ist eine Abwandlung des Indian Penal Code und galt seit 1871

31 Siehe dazu den höchst instruktiven Einblick in den Depeschenwechsel $z$ wischen dem Gouverneur Egerton und den Beamten des Kolonialministeriums bei Morris, a. a. O., S. $144 \mathrm{ff}$.

32 Lord Elgin, Secretary of State zu dieser Zeit, hatte das Gefühl, über die Geeignetheit der vorgeschlagenen Codes für Nigeria noch nicht hinreichend beraten zu sein und verschob die Entscheidung mit dem Hinweis auf die Einsetzung eines Komitees und eine Diskussion des Problems mit Lord Lugard, der zu jener Zeit Gouverneur von Nordnigeria war. Die Frage nach einem Strafgesetzbuch wurde vom südnigerianischen Gouverneur nicht wieder aufgegriffen.

33 Zur Kritik siehe Morris, a. a. O., S. 137. 


\section{Der Rechtszustand vor Einführung des Criminal Code 1904 bzw. 1916}

Wie in anderen von den Briten eroberten oder erworbenen Gebieten Afrikas, entwickelte sich auch in Nigeria nach der Niederlassung von Europäern ein Rechtsdualismus zwischen dem Recht und dem Gerichtssystem der eingeborenen Bevölkerung und dem der weißen Händler und Siedler.

2.1 Vor der Ankunft der Europäer hatte jeder Stamm sein eigenes Gewohnheitsrecht, das durch entsprechende Organe des Stammes, seien es die Emire und Alkali $^{34}$, seien es die Häuptlinge und ihr Rat von Unterhäuptlingen oder die Geheimgesellschaften ${ }^{35} \mathrm{bzw}$. Altesten- oder Familienhäupter ${ }^{36}$, angewendet wurde. Dieses allgemein anerkannte und von Stamm zu Stamm variierende Recht galt für die Eingeborenen in Lagos uneingeschränkt bis 1862, in den übrigen Gebieten des Landes bis zur Errichtung der Protektorate im Jahre 190037. Abgeändert und beeinflußt wurde das customary law und das an den ursprünglichen customary courts angewendete Verfahren durch englische Rechts- und Wertvorstellungen sowie durch die im Lande erlassenen Gesetze.

2.2. Mit der Ausdehnung der britischen Gerichtshoheit über das ganze Protektorat von 1900 an begann auch eine stärkere Einflußnahme auf und strengere Aufsicht der britischen Behörden über das Verfahren und die Anwendung des customary law in den ursprünglichen Gerichten. Einige mit den westlichen Humanitätsgrundsätzen nicht übereinstimmenden Maßnahmen wie Verstümmelung und Folter wurden verboten $^{38}$, Verfahren durch Gottesurteile ${ }^{39}$ und die Sklaverei, die eine beliebte gewohnheitsrechtliche Bestrafung rückfälliger Gewohnheitstäter war, endgültig abgeschaff $\mathrm{t}^{40}$.

Im übrigen durften das von den native courts angewendete Verfahren und die verhängten Strafen nicht den Grundsätzen von natural justice and humanity widersprechen ${ }^{41}$.

34 Im Norden gab es bei den Haussa/Fulani ein relativ gut entwickeltes Verwaltungs- und Rechtsprechungssystem. Oberster Richter und gleichzeitig Staatsoberhaupt war der Emir, der die wichtigsten Rechtsfälle selbst entschied. Es bildete sich aber schon bald ein selbständiger Richterstand heraus, der von Berufs wegen zur Rechtsanwendung herangezogen wurde: die Alkali.

35 In Westnigeria, im Bereich der Yoruba, gab es ein Exekutiv- und Rechtsprechungsorgan, den Oberhäuptling und seinen Rat von Unterhäuptlingen. Daneben hatten aber die Ogboni oder Geheimgesellschaften in wichtigen Angelegenheiten die Befugnis zur Entscheidung an sich gezogen.

36 Im Osten des Landes, wo es keine organisierten Staatshierarchien gab, sondern die Herrschaft von den Oberhäuptern der Clans oder Familien ausgeübt wurde, war der Rat der Altesten und der Familienoberhäupter für die Regelung wichtiger Rechtsfälle zuständig. Aber auch hier wurden häufig die Funktionen dieser Räte durch Geheimgesellschaften, zu denen theoretisch alle freigeborenen Männer der Gemeinschaft Zutritt hatten, ausgeübt. In der Praxis saßen in diesen Gesellschaften aber die pinflußreichen Persönlichkeiten und Familienoberhäupter.

37 Obwohl 1900 die britische Regierung "power and jurisdiction“ übernommen hatte, wurden die Gouverneure, die alleinige Gesetzgeber in den Protektoraten waren, durch Erlaß dazu angehalten ,to respect any native laws regulating the civil relations of any native chiefs, tribes or population under Her Majesty's protection... except so far as the same may be incompatible with the due exercise of Her Majesty's power and jurisdiction or clearly iniurious to the welfare of the said nations". Art. 6 der Southern Nigeria Order in Council, 1900 (zitiert nach Nwabueze, The Machinery of Justice in Nigeria, London, 1963, S. 4).

38 Native Courts Proclamation 1900, S. 9 (North); im Süden wurde ein entsprechendes Gesetz erst 1914 erlassen: Native Courts Ordinance 1914, S. 9.

39 Ordeal, Witchcraft and Ju-ju Proclamation 1903 (South), 1908 (North).

40 Slavery Proclamation. 1901 (North), Slaves Dealing Proclamation 1901 (South) in Verbindung mit Slavery Abolition Ordinance 1916.

41 Gewohnheitsrecht wurde in den englischen Gebieten Afrikas nur angewendet, wenn es den repugnancytest bestand, d. h. nicht gegen "Gerechtigkeit und bzw. oder Moral“ (Kenia, Malawi, Local Courts Ordinance 1962, S. 12) "natürliche Gerechtigkeit, equity und das gute Gewissen“ (Ghana bis 1960, Sierra Leone Court Act 1965, S. 76; Somaliland Constitution bis 1960), verstieß oder "mit den Grundsätzen der natürlichen Gerechtigkeit übereinstimmte" (Sierra Leone Local Court Act "1963, S. 2) usw. sätzen der natürlichen Gerechtigkeit übereinstimmte" (Sierra Leone Local Court Act 1963, S. 2) usw.
Siehe zu weiteren Formulierungen dieser Regel Allott, New Essays in African Law, 2. Aufl., London 1970, S. $158 \mathrm{ff}$. Der Begriff "natural justice“, aus dem englischen Recht stammend (siehe H. H. Marshall: Natural Justice, London 1959), umfaßt mehrere rechtsstaatliche Grundsätze, wie z. B.: Niemand soll Richter in eigener Sache sein; niemand soll verurteilt werden, ohne daß er angehört worden ist; dem Angeklagten sind die Einzelheiten der ihm zur Last gelegten Tat mitzuteilen; Entscheidungen sind zu 


\section{Der Rechtszustand nach Erlaß der Codes von 1904 bzw. 1916}

3.1 Gemäß der Präambel des 1904 in Nordnigeria eingeführten und 1916 in Südnigeria übernommenen Code war es das Ziel dieses Gesetzes „to declare consolidate and amend the criminal law". Dieses Bestreben bezog sich jedoch nur auf das in Nigeria geltende englische Recht; der bestehende Rechtspluralismus wurde mit der Inkraftsetzung des Code nicht beseitigt.

Der Rechtspluralismus war das Ergebnis der auch in anderen britischen Gebieten Afrikas praktizierten Rechtspolitik: Für die sich in der Kolonie Lagos ansiedelnden und im Protektorat lebenden Briten wurden britische Gerichte eingerichtet, an denen das englische Common Law bzw. die im Lande erlassenen Gesetze angewendet wurden. Außerhalb der Kolonie Lagos und insbesondere für die Masse der afrikanischen Einwohner galt weiterhin das Gewohnheitsrecht, obwohl in Südnigeria bereits in einem frühen Stadium der Kolonisation die Schwerkriminalität von englischen Gerichten nach englischem Recht abgeurteilt wurde. Grund für die Anerkennung und Duldung von native courts war der Mangel an britischen Richtern, außerdem der Wunsch nach Aufrechterhaltung der Ruhe in dem riesigen Gebiet, sowie die Bedingungen, unter denen die britische Justizhoheit erlangt worden war $^{42}$, wie auch die Überzeugung der Briten, daß ihr eigenes Recht zu weit entwickelt war, um auf eine Bevölkerung in einem weniger entwickelten Zustand angewendet werden zu können ${ }^{43}$. So führte denn auch die Einführung der Codes nicht zu einer Aufhebung dieses Dualismus. Die native courts durften kein Recht nach diesem Code sprechen; sie wandten weiterhin das Gewohnheitsrecht der Gegend an, soweit sie nicht ausdrücklich zur Anwendung einzelner Verordnungen oder Gesetze befugt waren ${ }^{44}$. Insbesondere in Nordnigeria lag trotz der Geltung des Code fast die gesamte Strafjustiz ${ }^{45}$ in den Händen der native courts. Islamisches Recht, obwohl schriftlich fixiert, galt in Nigeria als Gewohnheitsrecht ${ }^{46}$.

begründen; Strafen sollen nicht übermäßig hoch sein, sondern den Umständen der Straftat entsprechen. Die Begriffe Moral und gutes Gewissen sind wenig präzise und wurden in Afrika mit zunehmender Kenntnis der englischen Richter von den örtlichen Gegebenheiten und Bräuchen recht weit ausgelegt. Die Richter, die einen örtlichen Rechtsbrauch auf seine Anwendbarkeit hin prüfen mußten, lehnten aber solche Bräuche ab, die wirklich „barbarisch“ waren; sie gaben keine Begründungen für ihre Entscheidung, ergingen sich insbesondere "nicht in langen theoretischen Begriffsbestimmungen; vgl. Laoye v. Oyetunde (1944) A. C. 170, Eshugbayi Eleko v. Government of Nigeria (1931) A. C. 662. Tabitha Chiduku v. Chidamo, 1922 S. R.: „Whatever these words may mean, I consider that they should only apply to such customs as inherently impress us with some abhorrence or are obviously immoral in their incidence (zitiert nach Allott, New Essays, S. 162). Einige Beispiele aus Nordnigeria mögen den Einfluß der repugnancy-Klausel auf das Prozeßrecht verdeutlichen: Es verstößt nicht gegen die Grundsätze der natural justice, wenn ein Angeklagter zur Aussage als Zeuge gezwungen werden kann oder ein Zeuge zweimal aussagt oder keine zweite Zeugenaussage die Aussage des Komplizen bestätigt (Omaku v. Igbirra N. A. 1958 N. N. L. R. 11, C. A.); ein Verstoß hingegen ist es, wenn jemand wegen einer Straftat verurteilt wird, ohne daß eine Anzeige oder Anklage erhoben worden ist (Dzakpe v. Tif N. A. 1958 N. N. L. R. 135 C. A.). Auch verstößt es gegen natural justice, wenn der Angeklagte keine Zeugen zur Verteidigung nennen darf (Kano N. A. v. Obiara, 1960 N. N. L. R. 42, F. S. C.), siehe auch Park, The Sources of Nigerian Law, London, S. $68 \mathrm{ff}$.; weiterhin verstößt es gegen die rechtsstaatlichen Grundsätze, wenn ein wegen Straßenraubs Angeklagter an seiner Verteidigung gehindert wird (Guri v. Hadejia N. A. [1959] 4 F. S. C. 44).

42 Bei dem Erlaß von Gesetzen wurde in Nigeria die Formel wiederholt, die Lord Lugard gegenüber den Emiren der nördlichen Regionen als Bestandssicherung ihrer Rechte stets benutzte: "Nothing in any ordinance contained shall take away or affect any rights secured to any natives in The Protectorate by any treaties or agreements made on behalf or with the sanction of Her Late Majesty Queen Victoria, Her Heirs and successors and all such treaties and agreements shall be and remain operative and in force and all pledges and undertakings therein contained shall remain mutually binding." Royal Instruction 1946, Art. 17 (2). Siehe dazu Anderson, Islamic Law in Africa, London 1970, S. 173.

43 Allott, New Essays, S. $12 \mathrm{ff}$.

44 S. 4 des Code nahm native courts ausdrücklich von der Anwendung dieses Strafgesetzbuches aus: No person shall be liable to be trialed or punished in any court in Nigeria, other than a native tribunal, for an offence except under the express provision of the Code or some other ordinance.

$45 \mathrm{Ca} .90 \%$ : Anderson, Conflicts of Law in Northern Nigeria. 1959 I. C. L. Q., S. 446.

46 Allott (Hrsg.), The Future of Law in Africa. London 1960, Chapter II; Anderson, Islamic Law in Africa, Einleitung, S. 4; Okonkwo and Naish: Criminal Law in Nigeria. London 1964, S. 6. 
Da Lord Lugard, der im Norden Nigerias in den Emiraten ein hochentwickeltes Gerichtssystem vorfand, bei Errichtung des Protektorats den islamischen Herrschern zugesagt hatte, daß die britische Regierung sich in keiner Weise in die Ausübung der Religion des Landes einmischen werde, leiteten die Emire hieraus den Anspruch ab, ihr Recht - dieses als Teil ihrer Religion ansehend - selbst anzuwenden. So waren in Nordnigeria - anders als in Südnigeria, wo die britischen Gerichte sich die Aburteilung der schweren Kriminalität vorbehalten hatten - die Emire und ihre Alkali47 berechtigt, unter "native law and customs ${ }^{48}$ " Straftaten bis zur Schwere von Tötungsdelikten abzuurteilen und alle Strafen zu verhängen, die nicht wie Verstümmelung, Steinigung oder Kreuzigung verboten waren ${ }^{49}$. Dieses gleichberechtigte Nebeneinander zweier Rechtssysteme war einzigartig im Commonwealth; daß selbst für Tötungsdelikte zwei völlig unterschiedliche Rechtssysteme angewendet werden konnten, gab es in keinem anderen Land. Welches Rechtssystem anzuwenden war, hing davon ab, ob der native court für die Aburteilung des Angeklagten zuständig war, ob die Tat innerhalb des Bezirkes eines Emiratsgerichts begangen worden war, das für Tötungsfälle zuständig war, ob der Fall von der Nigerian Police oder der Native Authority Police aufgeklärt worden war und ob der dafür zuständige britische Verwaltungsbeamte den Fall von dem native court $z u$ einem englischen Gericht verwiesen hatte. Für den Angeklagten hing von der Beantwortung dieser Frage sehr viel ab, denn in manchen Fällen drohte der Code die Todesstrafe an, während das Maliki-Recht eine weniger schwere Strafe vorschrieb; in anderen Fällen konnte aber nach englischem Recht nur manslaughter (Totschlag) vorliegen, während nach islamischem Recht die Todesstrafe wegen Mordes zu verhängen war ${ }^{50}$. Dieser Rechtszustand beunruhigte die britische Administration, doch da das Maliki-Recht Teil der nordnigerianischen Lebensordnung war, wollte man aus politischen Gründen nicht mit drastischen Reformen vorgehen ${ }^{51}$.

Die 1933 durchgeführte Abänderung von s. 4 des Code - es wurden die Worte "other than a native tribunal" gestrichen - bedeutete keineswegs die Abschaffung des gewohnheitsrechtlichen Strafrechts ${ }^{52}$, vielmehr wurden lediglich die Strafbefugnisse der native courts dahingehend begrenzt, daß Körperstrafen von diesen Gerichten dann nicht verhängt werden dürfen, wenn sie im Code für das entsprechende Delikt nicht vorgeschrieben waren ${ }^{53}$.

Die Doppelspurigkeit im Strafrecht Nordnigerias, die zu zahlreichen Konflikten zwischen englischem und islamischem Recht führte, wurde auch nicht durch die englischen Rechtsmittelgerichte ausgeglichen. Diese prüften lediglich nach, ob die Strafsache nach dem angewandten Recht richtig entschieden worden war, befaßten sich jedoch nicht mit den Diskrepanzen zwischen englischem und Malikirecht,

47 Die von der Haussa gebrauchte Abwandlung des arabischen Wortes al-Quádi.

48 Das in Nordnigeria angewandte islamische Recht war eine leichte Abwandlung der Maliki-Form des Sharia-Rechts. Anderson, Islamic Law, S. 173.

49 Etwa der Vollzug der Todesstrafe durch das Schwert.

50 Das islamische. Recht kennt das Rechtsinstitut der Provokation nicht, bei deren Vorliegen nach englischem Recht eine Tötung nicht als Mord, sondern nur als Totschlag klassifiziert wird.

51 Okonkwo and Naish, Criminal Law, S. 6.

52 Criminal Code (Amendment) Ordinance 1933, S. 2

53 Native Courts Ordinance 1933, S. 10. Die Gesetzgebung von 1933 war Gegenstand widersprüchlicher Auslegung: im Falle Gubba v. Gwandu N. A. 1947 W. A. C. A. 141 wurde die Ansicht vertreten, S. 4 des Code hätte die Abschaffung des customary law bezweckt. Dem widersprachen die Berichte der Native Courts Commission of Inquiry 1949-1952. Zur Darstellung des Konflikts siehe Okonkwo and Naish, Criminal Law, S. $6 \mathrm{ff}$; sehr kritisch und ausführlich Milner, The Nigerian Penal System. London 1972, S. 24 f. 
überließen vielmehr die Verhinderung allzu krasser Ungerechtigkeit dem Gnadenrecht der Königin ${ }^{54}$.

Diese unerträgliche Situation wurde etwas abgemildert, nachdem in den Jahren 1946 und 47 der West African Court of Appeal mit zwei Fällen befaßt war, die wiederum das Problem der abweichenden Lösung von Tötungsfällen im islamischen und englischen Recht aufwarfen. Im Fall Bornu N. A. v. Magudama Abatcha entschied das Gericht, daß ein islamisches Gericht die Todesstrafe wegen einer Tötung verhängen könne, die nach englischem Recht nur als Totschlag gewertet würde; im Falle Gubba v. Gwandu N. A. jedoch wurde entschieden, daß die Strafe wegen einer Tat, die sowohl nach dem Code wie nach dem Gewohnheitsrecht mit Strafe bedroht war, nur noch den Vorschriften des Code zu entnehmen war ${ }^{55}$. Obwohl es nur wenige Taten gab, die nur nach customary law, nicht aber auch nach dem Code bestraft werden konnten, war diese Entscheidung nicht nur bedeutsam für das Recht der Tötungsdelikte, sondern gleichzeitig ein heftiger Schlag gegen die Herrschaft des Gewohnheitsrechts in Nordnigeria. Obwohl die native courts immer noch nur Gewohnheitsrecht anwenden durften, konnten sie doch keine höhere Strafe verhängen, als nach dem Code in demselben Fall hätte verhängt werden können ${ }^{56}$.

3.2 Die Vorbereitungen zur Erlangung der Unabhängigkeit verschärften die Unzufriedenheit mit dem gegenwärtigen Rechtszustand. Die Schwächen des Malikirechts ${ }^{57}$ ließen insbesondere die nichtmohammedanischen Bevölkerungsteile des Nordens fürchten, nach dem Weggang der britischen Beamten schutzlos der mohammedanischen Justiz ausgeliefert $\mathrm{zu} \operatorname{sein}^{58}$. Auch Überlegungen, daß ein

54 Anderson, Conflict of Laws in Northern Nigeria, 1957 J. A. L. S. 87 f. derselbe: Conflict of Laws in Northern Nigeria. 1959. I. C. L. Q., S. 445. Siehe auch den Fall Jalo T́samiya v. Bauchi N. A., 1957 N. R. N. L. R. 73 (81); eingehende Darstellung des Falles bei Anderson, Conflict of Laws in Northern Nigeria. 1957 J. A. L., S. $92 \mathrm{ff}$.

55 "We have therefore reached the conclusion that where a native court exercises its jurisdiction in relation to an act which constitutes an offence against the criminal code, whether or not it is also an offence against native law and custom it is required to exercise that jurisdiction in a manner not inconsistent, that is to say in accordance, with the provisions of the Code." Verity, C. J. in: Tsafo
Gubba v. Gwandu N. A. 1947, 12 W. A. C. A. 141. Siehe im einzelnen Okonkwo and Naish, Criminal Law, S. 8 f.; Anderson, Conflict of Laws in Northern Nigeria 1957 J. A. L. S. 88; zur Kritik am Fall Gubba und zur nachfolgenden Reaktion des Gesetzgebers: Milner, The Nigerian Penal System, S. $26 \mathrm{f}$.

56 Native Courts (Amendment) Ordinance 1951, s. 10 A; Native Courts Law 1956, s. 22 (North). Auch der Regionalisierung des Landes blieb dieser Grundsatz bestehen: Customary Courts Law 1956, s. 24 (East); Customary Courts Law (1959 Laws Chapter 31) s. 21 (West). Maizabo v. Sokoto N. A. 1957 (East); Customary Courts Law (1959 Laws Chapter 31) s. 21 (West). Maizabo v. Sokoto N. A. 1957
N. R. N. L. R. 133, F. S. C.; Jalo Tsamiya v. Bauchi N. A. 1957 N. R. N. L. R. 73; The position therefore is that $n$ while a native court may and indeed must ignore the provisions of the criminal code in trying a case under native law and custom, it must not impose a punishment in excess of the maximum punishment permitted for that act or omission under the criminal code or other enactment". Die Richter an den native courts hatten also zwei Prüfungen vorzunehmen: einmal mußten sie die Schuld nach customary law feststellen, danach die Tat nach dem Criminal Code prüfen, um das Strafmaß zu finden. Zur komplizierten Situation siehe Anderson, Conflict of Laws in Northern Nigeria. 1957 J. A. L. S. 97; 1959 I. C. L. Q. 442 ff. Siehe auch die kritische Anmerkung von Gower: Nigerian Statutes and Customary Law (1964). 1 N. L. J. $74 \mathrm{ff}$.

57 Anderson, Conflict of Laws in Northern Nigeria, 1959 I. C. L. Q. S. 442, 448: Politische Delikte waren nicht definiert und wurden von Alkali abgeurteilt, die von der native authority eingesetzt und daher abhängig waren; die Erben eines getöteten Mohammedaners konnten die Todesstrafe für den daher abhängig waren; die Erben eines getöteten Mohammedaners konnten die Todesstrafe für den
Angeklagten fordern, während die Tötung eines Nichtmohammedaners den mohammedanischen Tätern nicht mehr als die. Hälfte des Blutgeldes vom Wert eines Mohammedaners kostete, falls das Opfer ein Christ war, und ein Fünfzehntel der Summe, wenn der Getötete ein Heide war. Tötung des Täters konnten die nichtmohammedanischen Erben in diesen Fällen nicht verlangen, da das Leben eines Moslems viel mehr wert ist als das eines Nichtgläubigen. Auch im Beweisrecht wurden Unterschiede bezüglich des Wertes einer Aussage gemacht: Nichtmohammedanische Zeugen waren in Streitigkeiten, in denen eine Partei Moslem war, weitgehend nicht zeugnisberechtigt. Das Zeugnis von Frauen war in Strafsachen unzulässig. Tötungsfälle wurden bewiesen entweder durch außergerichtliches oder gerichtliches Geständnis oder durch das Zeugnis zweier Augenzeugen oder durch einen prima facie-Beweis gegen den Angeklagten, der von 50 Eidesleistungen der Blutsverwandten gestützt werden mußte, um darzutun, daß der Angeklagte des vorsätzlichen oder fahrlässigen Angriffs schuldig war und dieser Angriff kausal für den Tod des Opfers gewesen ist. Bei mehreren angeklagten Tätern war es den Blutserben überlassen, welchen der Angeklagten sie auf diese Weise töten lassen wollten, denn ihnen wurde nur ein Opfer zugestanden.

58 Bisher hatten britische Beamte die Befugnis, Fälle von native courts an englische Gerichte zu verweisen, was sie gewöhnlich in den Fällen taten, in denen zu befürchten war, daß unterschiedliche Religionszugehörigkeit von Täter und Opfer zur Ungleichbehandlung vor den Emiratsgerichten führen könnte. Auch blieb die Frage nach der Ausübung des Gnadenrechts ungelöst. 
unabhängiges Nigeria als Mitglied der Vereinten Nationen ein gewisses Maß an Rechtsstaatlichkeit nachzuweisen in der Lage sein sollte und als selbständiges Land auf ausländische Investitionen angewiesen war, die jedoch nur im Rahmen einer vertrauenerweckenden Rechtsordnung gemacht würden, führten schließlich dazu, daß Schritte unternommen wurden, das materielle und prozessuale Strafrecht des Nordens zu reformieren ${ }^{59}$. Nach Besuch mehrerer islamischer Staaten wurde eine internationale Juristenkommission gebildet ${ }^{60}$, die vorschlug, das Maliki Law, soweit es das Strafrecht betraf, aufzuheben und nur noch nach den Vorschriften eines Code zu richten. Dieses Gesetzbuch sollte nach dem Vorbild des Sudan Penal Code entworfen werden, obwohl vielleicht zu erwägen gewesen wäre, den auch im Norden seit 1904 geltenden Nigerian Criminal Code als Vorlage zu benutzen. Als Grund für die Wahl des Sudan Code wurden dessen leichtere Verständlichkeit für nichtausgebildete Richter, seine Bewährung in anderen mohammedanischen Ländern sowie die Tatsache, daß dem Criminal Code psychologische Hindernisse entgegenstünden, angeführt. So gilt seit 1. 10. 1960 - nach Überwindung nicht unbeträchtlicher Widerstände ${ }^{61}$ - im Norden der Penal Code $^{62}$, der das Gewohnheitsrecht ausdrücklich aufhob ${ }^{63}$. In der westlichen Region wurde die Abschaffung des customary law dadurch erreicht, daß den native courts die Zuständigkeit zur Aburteilung von Strafsachen ${ }^{64}$ entzogen wurde, und die Verfassung der Föderation enthielt den Grundsatz, daß „Verurteilung und Strafurteil wegen einer strafbaren Handlung nur aufgrund einer Tatbestandsdefinition und schriftlicher Strafbestimmung erfolgen könne ${ }^{65}$ “.

So wurde das dualistische System von Gewohnheitsrecht und gesetztem Recht in Nigeria ersetzt durch den Dualismus zweier unterschiedlicher Gesetzbücher. Für die Anwendung der Gesetze gilt Territorialitätsprinzip. Ein Nordnigerianer kann z. B. im Süden nicht wegen des einem Moslem gesetzlich untersagten Alkoholgenusses strafrechtlich verfolgt werden. Aus dem Territorialitätsgrundsatz ergeben sich die üblichen Probleme hinsichtlich des Begehungsorts, wenn einzelne Tatbestandsmerkmale in dem einen Bereich, andere im Geltungsbereich des anderen Gesetzes erfüllt worden sind. Beide Codes enthalten wörtlich übereinstimmende Regelungen dieser Frage ${ }^{66}$.

59 Siehe dazu den ausführlichen Bericht von Anderson, Conflict of Laws in Northern Nigeria. 1959 I. C. L. Q. S. $442,450 \mathrm{ff}$.

60 Die Mitglieder waren: der Chief Justice des Sudan, The Honorable Sayyid Muhamed Aburamat; der Vorsitzende des pakistanischen Law Reform Committee; Mr. Justice Muhamed Sharif, früher Richter am Supreme Court von Pakistan; der Waziri von Bornu, Chettima Kashim; Mr. Peter Achimugu, früherer Minister der Nordregion; Alkali Bida, Sheik Musa Othman und Professor Anderson, Professor of Oriental Laws und Direktor des Institute of Advanced Legal Studies der Universität London.

61 Siehe dazu Anderson, Conflict of Laws in Northern Nigeria. 1959 I. C. L. Q. S. 442, $450 \mathrm{ff}$.

62 No. 18 von 1959. Außer dem Penal Code 1959 gilt im Norden der Penal Code (Northern Region) Federal Provisions Act, 1960, der die Tatbestände enthält, deren Regelung der Bundesgesetzgebung vorbehalten war - also z. B. Staatsschutzdelikte, Zolldelikte, Münzdelikte, Straftaten gegen Post und Telegraphendienst.

63 Penal Code Law 1959 s. 3 (2): „No person is to be liable to punishment under any native Law or custom."

64 Customary Courts (Amendment) Law 1959 s. 9. (West).

65 Constitution of the Federation 1960 s. 21 (10); Republican Constitution 1963 s. 22 (9).

66 Criminal Code s. 12 A; Penal Code s. 4; siehe auch Beispiel bei Okonkwo and Naish, Criminal Law, S. 13. Diese Regelung gilt nicht, wenn Taterfolg der Tod eines Menschen ist und nur dieser Erfolg im Geltungsbereich eines der Codes eintritt, die Handlung aber im anderen Gebiet begangen wurde, in dem sich das Opfer zur Zeit der Tat aufhielt. Richardson, Notes on the Penal Code Law 2. Aufl. 1963, S. 11 


\section{Die Strafgerichtsbarkeit}

Dem Nebeneinander von customary law und englischem statute und common law bzw. in Nigeria erlassenem Gesetzesrecht, das ebenfalls auf englischem Recht beruhte, entsprach ein doppeltes Gerichtssystem, das sich in den verschiedenen Landesteilen unterschiedlich entwickelte.

\section{Die britischen Gerichte}

1.1 Für die sich in der Kolonie Lagos ansiedelnden Europäer wurden nach einer Phase der Konsulargerichtsbarkeit ${ }^{67}$ britische Gerichte ${ }^{68}$ eingerichtet, von denen die im Lande erlassenen Gesetze ${ }^{69}$ sowie englische Gesetze ${ }^{70}$, die auch in Nigeria galten, und common law ${ }^{71}$ angewendet wurden.

1.2 Mit der Ausdehnung der britischen Herrschaft ins Landesinnere, die 1900 mit der Erklärung der Protektorate Nord- und Südnigeria ihren einstweiligen Abschluß fand, erhielten die Briten auch die Jurisdiktionsgewalt in diesen Gebieten ${ }^{72}$, soweit nicht Sonderverträge dieses Recht ausschlossen ${ }^{73}$. Aber selbst in diesen Ausnahmefällen (Yorubaland und Egbaland) schlossen die Briten mit den Stammesführern Einzelverträge, durch die ihnen die Zuständigkeit für Aburteilung von Nichteingeborenen wegen anklagbarer (also schwerer) Delikte zugestanden wurde. In Egbaland war ein gemischtes Gericht, besetzt mit Briten und Egbas zuständig für die leichtere Kriminalität der Europäer; für die Tötungsdelikte wurde die Zuständigkeit über alle Einwohner, also sowohl Europäer als auch Eingeborene, auf den Supreme Court in Lagos übertragen ${ }^{74}$. Im Falle der Yorubas waren die britischen Gerichte zuständig für alle Straftaten, die von Europäern begangen wurden, außerdem bei Tötungsdelikten auch über die eingeborene Bevölkerung ${ }^{75}$.

In dieser Politik zeigte sich das große Interesse der Briten, sogar noch vor Erhalt der politischen Herrschaft, spätestens aber gleichzeitig mit der Machtausübung die Justizhoheit zu erlangen, die sich möglichst nicht nur auf die Europäer in einem bestimmten Landesteil erstrecken sollte, sondern in den Fällen schwerer Kriminalität auch die eingeborene Bevölkerung miteinbezieht.

Alle diese Gebiete Westnigerias unterstanden dem einzigen britischen Gericht in Lagos, dem sogenannten Supreme Court, der mit dem Chief Justice und einer

67 Siehe Nwabueze, The Machinery of Justice in Nigeria, S. $47 \mathrm{ff}$. und $51 \mathrm{ff}$.

68 In Lagos, das 1862 zur Kolonie erklärt wurde, errichtete die britische Regierung alsbald ordnungsgemäß funktionierende Gerichte; der Chief Magistrate's Court von 1863 wurde 1866 abgelöst durch den Court of Civil and Criminal Justice, der seinerseits 1875 durch ein oberstes Gericht mit umfassender Zuständigkeit ersetzt wurde, den Supreme Court. Siehe Supreme Court Ordinance, 1876.

69 Zur Rechtssetzungsbefugnis in der Kolonie Lagos und in den Protektoraten siehe oben S. 225.

70 Englische Gesetze galten in Nigeria entweder, weil sie von allgemeiner Bedeutung waren und für das ganze Empire erlassen wurden: z. B. der Foreign Jurisdiction Act 1890; oder weil sie von der örtlichen Gesetzgebung freiwillig übernommen wurden, da diese nicht auf allen Rechtsgebieten eigenständige Gesetze erlassen konnten. Vgl. Nwabueze, The Machinery of Justice in Nigeria, S. 17.

71 Common Law enthielt den überwiegenden Teil des englischen Rechts zu Anfang des Jahrhunderts.

72 Die britische Kolonialregierung in Lagos hatte die Befugnis erhalten (Imperial Orders in Council 1887 und 1901) "power und jurisdiction“ in den erworbenen Landesteilen auszuüben.

73 Ausgenommen war zunächst Yorubaland, in dem die vollständige Herrschaft noch nicht errichtet war; auch Egbaland hatte noch seine Unabhängigkeit bewahrt. Beide Gebiete waren aber bis 1915 in das Protektorat Südnigeria einbezogen worden. Damit war auch in diesen Gebieten die britische Gerichtshoheit begründet. Jurisdiction of Courts extension (Protectorate) Ordinance 1915.

74 Agreement vom 8. August $1904 \mathrm{zwischen} \mathrm{der} \mathrm{englischen} \mathrm{Krone} \mathrm{und} \mathrm{den} \mathrm{Machthabern} \mathrm{von} \mathrm{Egbaland;}$ Egba (Jurisdiction of Supreme Court) Ordinance 1904.

75 Z. B. Ibadan und Oyo Jurisdiction of the Supreme Court Ordinance, 1904. Zur traditionellen Gerichtsbarkeit im Yorubaland - heute Western Nigeria - siehe: Kayode: Judicial Administration in a Changing Society - Customary Courts in W'estern Nigeria, „VRU“ Bd. 8 (1975), S. 435 ff. 
kleinen Zahl von Richtern völlig unzureichend ${ }^{76}$ besetzt war. Das Gericht entsprach dem englischen High Court und bestand aus folgenden Organen:

a) Als unterste Instanz judizierten die sog. District Courts mit beschränkter Zuständigkeit: Ihre Strafgewalt ging bis zu sechs Monaten Gefängnis, 15 Stockhieben oder 50 £ Geldstrafe. Besetzt waren sie mit dem Verwaltungsbeamten des Bezirks, der kraft Amtes Commissioner des Supreme Court war.

b) Unbeschränkte zivile und strafrechtliche Zuständigkeit hatten die mit einem Richter besetzten Divisional Courts, die gleichzeitig Rechtsmittelgericht für den District Court waren.

c) Die Spitze des Gerichtsaufbaus im Süden bildete der Full Court mit Sitz in Lagos, besetzt mit zwei oder mehr Richtern des Supreme Court und dem Chief Justice als Vorsitzenden ${ }^{77}$. Rechtsmittel gegen Entscheidungen des Full Court waren an den Privy Council in London zu richten ${ }^{78}$.

1.3 Von dem südnigerianischen Gerichtswesen unterschied sich die britische Justiz im Norden des Landes durch zahlreiche Abweichungen. Aus grundsätzlichen Erwägungen und aus Gründen der Schnelligkeit, Wirtschaftlichkeit und Bequemlichkeit hatte Lord Lugard das System der Rechtsprechung durch Verwaltungsbeamte eingeführt, von dem er sich stärkere Berücksichtigung des Gewohnheitsrechts versprach. Verwaltungsbeamte waren seiner Meinung nach nicht so stark formalrechtlich fixiert wie Juristen, kannten die Verhältnisse in ihrem Bezirk besser und waren eher bereit, sich in Zeiten politischer Schwierigkeiten über rein juristische Bedenken hinwegzusetzen ${ }^{79}$.

Es gab im Norden zwar auch einen Supreme Court ${ }^{80}$, doch war dessen örtliche Zuständigkeit auf bestimmte Gebiete begrenzt ${ }^{31}$. In diesen Gebieten war er für alle Bevölkerungsteile zuständig; in den übrigen Gebieten hatte er die Gerichtshoheit nur über Nichteingeborene und solche Eingeborenen, die in Regierungsdiensten standen, sowie bei zivilen Straftaten von Militärangehörigen.

Neben dem begrenzt zuständigen und deshalb recht unbedeutenden Supreme Court gab es den Provincial Court ${ }^{82}$, der unbegrenzte Zuständigkeit in zivil- wie in strafrechtlichen Sachen hatte. Richter dieses Gerichts war der Resident der Provinz, also der höchste Verwaltungsbeamte des Gebiets. Weitere Richter waren die untergeordneten Verwaltungsbeamten als Commissioners des Gerichts kraft Amtes. Anwälte waren an diesem Gericht nicht zugelassen; Rechtsaufsicht führte der High Commissioner des Protektorats, also auch ein Laie, der sich jedoch von Juristen beraten ließ. Er mußte Strafen wie Todesstrafe, Deportation, Gefängnis über 6 Monaten, Stockstrafen über 12 Schläge und Geldstrafen über $50 £$ bestätigen.

\footnotetext{
76 Nwabueze, The Machinery of Justice in Nigeria, S. 60; und die dort S. 61 wiedergegebene Kritik des Chief Justice Speed: "The activities of the Court were circumscribed by the fact that its organization was faulty. It was never adquately manned... Its staff was to small, and its organiziation so rudimentary, that outside the grading centers and closed towns its criminal jurisdiction
was rarely exercised, ..."
Es war also durchaus üblich, daß derselbe Richter in erster und zweiter Instanz an der Ent7 Es war also durcha scheidung mitwirkte.

78 Appeals to the Privy Council (Southern Nigeria) Order in Council, 1909.

79 Lugard, The Dual Mandate, S. 539.

80 Errichtet durch die Protectorate Courts Proclamation, - Act No. 4 of 1900; Supreme Court Proclamation, 1902.

81 Sogenannte cantonments und die Provinzen Niger, Ilurin, Kabba, Bassa, Kantagora und Nassarawa.

82 Errichtet durch die Protectorate Courts Proclamation, - Act No. 4 of 1900; Provincial Court Proclamation 1902.
} 
1.4 Mit der Vereinigung von Süd- und Nordnigeria zum Protektorat Nigeria wurde das Gerichtswesen des Nordens mit leichten Abänderungen auch im Süden eingeführt. Die territoriale Zuständigkeit des Supreme Court wurde auf die Townships begrenzt ${ }^{83}$, wo er jedoch für alle Bewohner zuständig war. Außerhalb der Townships gab es konkurrierende Zuständigkeit des Supreme Court und der Provincial Courts über Nichteuropäer. Erst 1933 wurde das Justizwesen reformiert: Die Provincial Courts, Gegenstand jahrzehntelanger Kritik ${ }^{84}$, wurden dank der energischen Initiative des Gouverneurs Cameron abgeschafft, die Zuständigkeit des Supreme Court auf Lagos beschränkt, wo er für alle Personen und alle Streitigkeiten unbegrenzt zuständig war ${ }^{85}$, und im Protektorat wurden sogenannte Protectorate Courts geschaffen, die High Courts und die Magistrates' Courts ${ }^{86}$. Rechtsmittel vom High Court gingen nicht zum Supreme Court Lagos, sondern zum West African Court of Appeal, der auch Rechtsmittelinstanz für den Supreme Court war ${ }^{87}$.

Die Protectorate Courts bewährten sich nicht und wurden 1943 beseitigt. An ihre Stelle trat nun der Supreme Court ${ }^{88}$ mit unbeschränkter zivil- und strafrechtlicher Gerichtsbarkeit im ganzen Land. Er war gleichzeitig Rechtsmittelinstanz für die Magistrate's Courts. Der West African Court of Appeal bestand fort ${ }^{89}$.

Mit der Verfassungsänderung 1954, durch die Nigeria in drei Regionen und das Gebiet von Lagos aufgeteilt wurde, wurden in jeder Region eigene Gerichtszüge aufgrund regionaler Gesetzgebung geschaffen. Dieser Gerichtsaufbau besteht auch heute noch und gilt, mit einzelnen Abwandlungen, in allen 12 Staaten des Landes. Die untere Instanz der Strafgerichtsbarkeit bilden die Magistrates' Courts ${ }^{90}$, die seit 1933 die Lücke im Justizsystem zwischen dem High Court mit einem komplizierten Verfahren und den formlos judizierenden customary courts bildeten. Die an diesen Magistrates' Courts tätigen Richter sind - anders als in England keine Laien, sondern ausgebildete Richter. Im formlosen summarischen Verfahren erledigen sie die Mehrzahl der leichten und mittelschweren Strafsachen und führen auch die Voruntersuchung in Fällen schwerer Verbrechen, deren Aburteilung dem High Court vorbehalten ist. Die höchste Stufe der Magistrates' Courts kann Freiheitsstrafen bis zur Dauer von fünf Jahren oder Geldstrafen bis zur Höhe von 500 £ verhängen.

83 Townships waren Sitz einer Regierungseinrichtung in einer Eingeborenenstadt. Die Bevölkerung dieses Viertels war wegen der Beamten und Regierungsbediensteten gemischt, zog auch zahlreiche Händler und Gewerbetreibende an. Es gab 6 Townships im Norden und 52 im Süden. Township Ordinance, 1917.

84 Nwabueze, The Machinery of Justice in Nigeria, S. 65.

85 The Supreme Court (Amendment) Ordinance 1933.

86 Theoretisch hatte allerdings der Supreme Court auch unbegrenzte strafgerichtliche Zuständigkeit im Protektorat über Nichteingeborene, und in den Townships auch über Eingeborene. Er ließ diese Gerichtsbarkeit jedoch durch die High Courts ausüben. Siehe Nwabueze, The Machinery of Justice in Nigeria, S. 66. Grund für die Errichtung einer zweiten Art von Gerichten im Protektorat war der Mangel an erstklassigen Richtern, die am Supreme Court hätten beschäftigt werden können. Man mußte daher ein weniger anspruchsvolles Gericht im Lande gründen, anstatt die Zuständigkeit des Supreme Court auf das ganze Land auszudehnen.

87 Der West African. Court of Appeal ersetzte somit den früheren Full Court des Supreme Court. Er war besetzt mit Richtern der Supreme Courts der westafrikanischen Gebiete. Rechtsmittel gegen seine Entscheidungen gingen an den Privy Council in London. West African Court of Appeal Ordinance 1933; West African (Appeal to the Privy Council) Order in Council 1930.

88 Supreme Court Ordinance 1943.

89 Der West African Court of Appeal bestand seit 1928; erst 1933 wurde er auch für Rechtsmittel aus Nigeria zuständig. West African Court of Appeal Ordinance, 1933.

90 Magistrates' Court (Lagos) Act, cap. 113 Laws of the Federation; Magistrates' Courts Law, 1955 Western Region; Magistrates' Courts Law, 1955 - Eastern Region; Criminal Procedure Court Law, 1960 - Northern Region. Zur Besetzung und Einteilung der Gerichte siehe Nwabueze, The Machinery of Jutice in Nigeria. S. $136 \mathrm{ff}$.; Ezejiofor, The Federation of Nigeria, a. a. O., S. $47 \mathrm{ff}$. 
Die Spitze der Gerichtsbarkeit jeder Region bzw. heute jedes Staates ist der High Court, der die gleiche Funktion wie der landeseinheitliche Supreme Court der Jahre 1943-1955 ausübt ${ }^{91}$. Dieses Gericht ist ein court of equity ${ }^{92}$, d. h. es kann auch in Strafsachen auf eine Versöhnung zwischen Opfer und Täter hinwirken und zur Streitschlichtung ermuntern, sofern es sich um Vergehen, insbesondere Körperverletzungsdilikte, handelt und keine erschwerenden Umstände vorliegen ${ }^{93}$. Leider ist nicht bekannt, ob die Gerichte von dieser Befugnis, die ihrem Wesen nach Ausdruck alt-afrikanischer Rechtsideen ${ }^{94} \mathrm{zu}$ verwirklichen sucht, Gebrauch machen $^{95}$.

Die Bedeutung der High Courts ist groß, da sie die einzigen höheren Gerichte der ersten Instanz in jeder Region sind und alle wichtigen Strafsachen vor diesem Gericht verhandelt werden. Als Rechtsmittelgericht ist der High Court zuständig für die Anfechtung der Urteile der Magistrates' Courts. Er überwacht die Rechtsprechung der unteren Gerichte weiterhin mittels der sog. revision: die Magistrates' Courts (und in Westnigeria auch die customary courts) müssen dem High Court jeden Monat die erledigten Strafsachen zur Prüfung vorlegen. Der High Court kann im Fall unrichtiger Entscheidungen dann seinerseits alle Anordnungen treffen, die ihm gerecht und zweckmäßig erscheinen ${ }^{96}$.

An der Spitze der gesamtnigerianischen Gerichtsbarkeit steht der Federal Supreme Court ${ }^{97}$, der seinen ständigen Sitz in Lagos hat, aber - jedenfalls früher - auch in den Regionen zusammentrat, um die Verbindung zu den inneren Teilen des Landes zu behalten. Das Gericht besteht aus dem Chief Justice des Landes und 8 Richtern ${ }^{98}$. Der Supreme Court war in erster Linie Verfassungsgericht ${ }^{99}$; im Bereich der Strafgerichtsbarkeit ist der Supreme Court höchste Rechtsmittelin$s \tan z^{100}$ für die Anfechtung von Urteilen der regionalen High Courts ${ }^{101}$.

91 Siehe High Court Law 1955 für Western, Eastern, Northern Nigeria sowie den High Court of Lagos Act, cap. 80 Laws of the Federation. Zu Einzelheiten siehe Nwabueze, The Machinery of Justice in Nigeria, S. $156 \mathrm{ff}$.

92 Als court of equity kann das Gericht Billigkeitsentscheidungen treffen.

93 High Court of Lagos Act, Cap. 90, s. 26; High Court Law (Western), s. 25; High Court Law (Eastern), s. 31. Siehe auch Magistrates' Courts (Lagos) Act s. 24; Magistrates' Courts Law, s. 35 (Western). Diese Befugnis zur Streitschlichtung und Aussöhnung findet sich auch in anderen Rechtsordnungen Afrikas: z. B. Criminal Procedure Code (Laws of Tanganjika cap. 20), s. 134; Crimanal Procedure Code (Laws of Kenya cap. 75), s. 176; Criminal Procedure and Evidence Code (Laws of Malawi cap. 8:01), s. 161.

$94 \mathrm{Ziel}$ der afrikanischen gewohnheitsrechtlichen Streitschlichtung war die Aussöhnung der Streitenden und die Heilung der durch die Begehung einer Straftat in der Gemeinschaft entstandenen Streitsituation.

95 Skeptisch dazu: Nwabueze, The Machinery of Justice in Nigeria, S. $161 \mathrm{f}$. ; und Milner, Nigerian Penal System, S. 133

96 Siehe Nwabueze, The Machinery of Justice in Nigeria, S. $173 \mathrm{ff}$.

97 Nigeria (Constitution) Order in Council, 1960 ss. 104-113 i. d. F. Constitution of the Federation Act, 1963 , s. 111. Seit dieser Verfassung heißt das Gericht nur noch Supreme Court. Federal Supreme Court Act, 1960; Federal Supreme Courts Rules. L. N. 96 von 1961.

98 Justices of the Supreme Court Act, 1964, s. 1.

99 Die Zuständigkeit zur Entscheidung von Streitigkeiten zwischen Einzelstaaten oder zwischen der Bundesregierung und den Regierungen der Einzelstaaten wurde ihm mit Decree No. 1 von 1966 (Suspended Provisions of Constitution of the Federation) genommen.

$100 \mathrm{Die}$ republikanische Verfassung verbot jedes Rechtsmittel zu anderen Gerichten (s. 120). Rechtsmittel zum Privy Council wurden damit abgeschafft (Constitution of the Federation 1963, s. 158 (3)). Der West African Court of Appeal hatte schon 1955 aufgehört, Rechtsmittelinstanz für Nigeria zu sein, als der damalige Supreme Court geschaffen wurde.

101 Eine Ausnahme bildet der Western State, der über dem High Court noch einen Court of Appeal hat, gegen dessen Urteile erst Rechtsmittel zum Supreme Court eingelegt werden kann: Constitution (Miscellaneous Provisions) (No. 2) Decreee 1967; Court of Appeal Edict, 1967; Constitution (Western Nigeria Court of Appeal) Order 1967 


\section{Die afrikanischen Gerichte}

Zunächst waren die native courts tatsächlich traditionelle Eingeborenengerichte, also Organe, die den ursprünglichen Bewohnern in ihrer traditionellen Form als Schlichtungsstätte für Streitigkeiten dienten. Ab 1900 begannen aber die Briten sog. statutory native courts einzurichten, die in Lagos, Süd- und Nordnigeria unterschiedlich organisiert waren.

2.1 Die Einteilung des Südens in sog. Native Court Areas, in denen jeweils ein solches Gericht seinen Sitz hatte, folgte keinen Stammesgrenzen oder kulturellen Einheiten. Es gab ein höheres (Native Council) und ein niederes (Minor Court) Gericht, deren Zuständigkeit auf Eingeborene beschränkt war ${ }^{102}$. Diese Gerichte stellten - im Widerspruch zu ihrer Bezeichnung - nun jedoch keineswegs das traditionelle Eingeborenengericht dar oder setzten es fort. Der Native Council bestand aus dem höchsten Verwaltungsbeamten (District Commissioner) als Vorsitzendem und ernannten Vertretern der umliegenden Dörfer oder Städte. Diese Vertreter waren aber nicht die traditionellen Führer, sondern Personen, die wegen ihrer Geneigtheit zur Zusammenarbeit mit den Europäern dem Regierungsbeamten aufgefallen waren ${ }^{103}$. Die Afrikaner waren nur Beisitzer, obwohl das Urteil in ihrem Namen erging. Sie wurden nur herangezogen, um dem Vorsitzenden in Fragen des Gewohnheitsrechts zu helfen, das jedoch nicht allzu oft angewendet wurde, da der Vorsitzende seine eigene Version des englischen Rechts bevorzugte ${ }^{104}$. Kennzeichnend für den Charakter dieser Gerichte ist eine richterliche Beschreibung: "Native Courts are not native institutions at all. They are creatures of statutes of the Nigerian Government. Under the Native Courts Ordinance a Native Court needs not necessarily even consist of natives. It seems to me obvious that there can be no native law and custom dealing with a procedure of an institution which is the creature of what in this connection I may call foreign statutes of the last thirty years also and has no place in native law and custom ${ }^{\mathbf{1 0 5}}$." Ihre Strafgewalt für die ca. acht Vergehen, für die sie zuständig waren ${ }^{106}$, betrug bis zu einem Jahr Gefängnis oder $100 £$ Geldstrafe bzw. als Minor Court: sechs Monate Gefängnis und 50 £ Geldstrafe ${ }^{107}$. Das Gericht konnte außerdem traditionelle Strafen verhängen, sofern diese nicht der natürlichen Gerechtigkeit oder den englischen Rechtsgrundsätzen widersprachen. Diese durch Gesetz eingerichteten Gerichte besaßen im Gegensatz zu den früheren traditionellen native courts einen Vollstreckungsapparat, insbesondere gab es Gefängnisse, eine für Afrikaner neuartige Einrichtung, da Freiheitsentzug bisher als Sanktion in Afrika unbekannt war ${ }^{108}$.

102 Mit Zustimmung des Betroffenen konnte an diesen Gerichten auch gegen einen Europäer verhandelt und entschieden werden; dies kam jedoch nie vor. Nwabueze, The Machinery of Justice in Nigeria, S. 71.

103 Nwabueze, The Machinery of Justice in Nigeria, S. 70.

104 Nwabueze, The Machinery of Justice in Nigeria, S. 70.

105 Graham Paul, J. in: (1936) 13 N. L. R. 30.

106 Leichte Körperverletzung, Verzauberung mittels eines Fetischs, vorsätzlicher Ungehorsam oder Nichtbefolgung des Befehls eines Familienoberhauptes, Besitz vergifteter Gegenstände mit der Absicht, diese zur Gefährdung des Lebens eines anderen zu benutzen, vorsätzliche Brandstiftung oder Sachbeschädigung.

107 Hatte der District Commissioner den Vorsitz beim Minor Court, erhöhte sich die Strafgewalt und entsprach der des Native Council.

108 Milner, The Nigerian Penal System, S. 34 mit Nachweisen ethnologischer und anthropologischer Literatur. 
2.2 Im Gegensatz zum Süden wurden im Norden die traditionell bestehenden Gerichte übernommen ${ }^{109}$; es gab drei Instanzen:

a) das Gericht des Emirs, bestehend aus ihm und einigen Mitgliedern seines Rates, die als Beisitzer fungierten;

b) das Gericht der Alkali mit diesen Berufsrichtern als einzigen Richtern;

c) in den nicht islamischen Gebieten wurden je nach der politischen Struktur Häuptlingsgerichte oder Gerichte mit ernannten „Richtern“ eingeführt.

An den islamischen Gerichten wurde das Maliki-Recht angewendet ${ }^{110}$, dessen Strafen außer Verstümmelung oder Folter verhängt und vollzogen werden durften, solange sie nicht gegen die natural justice oder die Humanitätsvorstellungen der Briten verstießen. Das Oberste Gericht, der Emir's Court, konnte auch die Todesstrafe verhängen, zu seiner Zuständigkeit gehörte auch die Schwerkriminalität. Todesurteile mußten allerdings vor der Vollstreckung vom Gouverneur bestätigt werden. Personell waren diese native courts des Nordens zuständig für alle Eingeborenen außerhalb der sog. cantonments, Gebieten, in denen die britischen Gerichte zuständig waren ${ }^{111}$. Ausgenommen von der Gerichtsbarkeit waren zudem die in Regierungsdiensten stehenden Eingeborenen.

2.3 Nach dem Zusammenschluß von Nord- und Südnigeria zum Protektorat Nigeria wurde das nördliche Prinzip der Aufrechterhaltung traditioneller Eingeborenengerichte auch im Süden durchgeführt ${ }^{112}$. Der District Officer hörte auf, vorsitzende Richter des native court zu sein, und in den Gebieten, in denen es eine funktionierende traditionelle Gerichtsbarkeit gab ${ }^{113}$, insbesondere also im Westen, wurden die Häuptlingsgerichte wieder anerkannt, bekamen aber nicht unbegrenzte Zuständigkeit verliehen, wie sie die nördlichen traditionellen Gerichte hatten; die Todesstrafe durfte von diesen Gerichten nicht verhängt werden. Im Osten konnte auf frühere Gerichtstradition durch die Häuptlinge nicht zurückgegriffen werden. Hier blieben an den Gerichten, die meist nur ganz geringfügige Zuständigkeit hatten, die sogenannten warrant chiefs, also die von den Engländern in Ermangelung traditioneller Häuptlinge eingesetzten Eingeborenengerichtsorgane14. Erst mit den Reformen von 1933, angeregt und durchgeführt durch den damaligen Gouverneur Cameron, wurde auch hier auf die traditionellen Gerichte in Form von Ältesten- und Familienoberhäupterräten zurückgegriffen.

Für den Norden und Westen brachten die Reformen eine Einschränkung ihrer Strafgewalt und eine Modifikation ihrer Unabhängigkeit von britischen Gerichten $^{115}$.

109 Native Courts Proclamation 1906. Vorher war ein Versuch gemacht worden, durch den Obersten Verwaltungsbeamten mit Zustimmung des traditionellen Herrschers, des Emirs, native courts einzurichten (Native Courts Proclamation 1900). Der Versuch scheiterte jedoch.

110 Maliki-Recht ist das islamische kodifizierte Recht, wie es von der Maliki-Schule abgewandelt worden war.

111 Siehe oben S. 234

112 Native Courts Ordinance 1914.

$113 \mathrm{Im}$ Westen des Landes hatten die traditionellen Rechtsprechungsorgane auch in der Zeit von 1900 bis 1914, als die gesetzlich errichteten Native Courts offizielle Gerichte für die Eingeborenen waren, eine inoffizielle Rolle im Untergrund gespielt. Sie wurden von den Verwaltungsbeamten teils als wertvoll geduldet, teils mit Strafe bedroht, weil hier Rechtsfälle außerhalb der dafür vorgesehenen Organe entschieden wurden. Ihr Vorteil wurde insbesondere in der geringeren Anfälligkeit der Rechtsprechenden für "Aufmerksamkeiten" und in der örtlichen Nähe der Gerichte zu den Rechtssuchenden gesehen. Das "weniger förmliche Verfahren entsprach auch stärker den Vorstellungen der ländlichen Eingeborenen. Vgl. Report on Native Courts; Bericht einer Kommission über die Eingeborenengerichtsbarkeit in den drei Regionen. Government Printer Kaduna 1952. Zitiert nach Nwabueze, The Machinery of Justice in Nigeria, S. 76. S. auch Kayode: Judicial Administration in a Changing Society - Customary Courts in Western Nigeria, in „VRÜ“ Bd. 8 (1975), S. $438 \mathrm{ff}$.

114 Zur Geschichte, Funktion und Kritik an diesen Institutionen s. Native Courts Report (Eastern Region), 1953.

115 Siehe dazu oben S. 230. 
Mit der Aufteilung in drei Regionen und das Gebiet Lagos im Jahre 1956 wurden auch die customary courts regionalisiert. Jede Region errichtete ihre eigenen customary courts, deren Zuständigkeit durch Gesetz bzw. durch die Errichtungsverordnungen (warrants) festgelegt wurde116. Diese Gerichte waren im Norden zuständig für "persons and classes of persons who have ordinarily been subject to the jurisdiction of native tribunals" sowie Personen, die die allgemeine Lebensweise der Landbevölkerung angenommen hatten ${ }^{117}$. Die in fünf Grade ${ }^{118}$ eingeteilten native courts hatten umfassende Strafgewalt: die Gerichte der Kategorie A konnten die Todesstrafe verhängen ${ }^{119}$; die Gerichte der Kategorie A-limited waren für alle Schwerkriminalität mit Ausnahme der Tötungsdelikte zuständig, die Gerichte der Kategorie B konnten Gefängnisstrafen bis zu drei Jahren oder Geldstrafen bis 150 \& verhängen, während Kategorie $C$ und $D$ für die leichtere Kriminalität zuständig waren ${ }^{120}$. Außerdem konnten aus dem Gewohnheitsrecht übernommene Strafen wie Stock- oder Peitschenschläge verhängt werden ${ }^{121}$. Rechtsmittel gegen die Entscheidungen der Gerichte der Kategorie A und A-limited sowie der Provincial Courts konnten zur Native Courts Appellate Division des High Court eingelegt werden; für Rechtsmittel gegen Urteile der Gerichte B, C und D war der Provincial Court ${ }^{122}$ zuständig.

Seit der Neugliederung des Nordens in sechs Staaten ${ }^{123}$ ist auch das Gerichtswesen noch stärker regionalisiert worden; das Area Courts Edict von 1967 löste das Native Courts Law 1956 ab und ersetzte die Native Courts durch sog. Area Courts. Dies war das Ergebnis langandauernder Kritik an den Native Courts, denen vorgeworfen wurde, sie würden von den örtlichen Behörden, denen sie unterstanden, zum Mißbrauch ihrer Amtsgewalt und zu Schikanen benutzt ${ }^{124}$. Die Area Courts waren nicht mehr wie die Native Courts Teil der Eingeborenenverwaltung und damit deren Einfluß stark ausgesetzt, sondern wurden jetzt auch verwaltungsmäßig der Judikative zugeordnet. Geschaffen werden die Area Courts durch den Chief Justice; alle Richter dieser Gerichte sind von 1967 an Staatsbeamte. Die unteren Area Courts sind eingeteilt in drei Kategorien (I, II und III); die höheren Area Courts sind Rechtsmittelinstanz und Eingangsinstanz für Strafsachen, deren Gegenstand schwerere Delikte sind. Seit Einführung des Nigerian Penal Code dürfen diese Gerichte, wie schon vorher die Native Courts, das MalikiRecht nicht mehr anwenden. Einzige Grundlage des Strafrechts ist heute auch für die Area Courts der Nigerian Penal Code und andere im parlamentarischen Verfahren erlassene Gesetze. Das Verfahren hat sich nach einer Übergangsphase, in der

116 Native Courts Law 1956 (Northern Region); Customary Courts Law 1956 (Eastern Region); Customary Courts Law 1957 (Western Region).

117 s. 15 Native Courts Law 1956. Zur Interpretation und Kritik der Vorschrift siehe Keay and Richardson: The Native and Customary Courts of Nigeria. London 1966, S. $170 \mathrm{ff}$. Ausgenommen von der Gerichtsbarkeit der native courts waren nach dieser Allgemeinregelung die aus Lagos, Port Harcourt oder anderen Teilen der Küstenregion stammenden Einwohner des Nordens, soweit sie sich nicht in ihrer Lebensweise den dort wohnenden Nordländern angepaßt hatten, sowie auch nördliohe Bewohner, die praktizierende Christen waren, gebildete Einwohner oder solche, die einen westlichen Lebensstil führten.

118 A, A-limited, B, C und D.

119 Zur Vollstreckung der Todesstrafe mußte jedoch die Bestätigung des Urteils durch den Minister eingeholt werden.

120 C: Gefängnis bis zu 18 Monaten oder Geldstrafe bis zu £ 30; D: Gefängnisstrafe bis zu 9 Monaten oder Geldstrafe bis zu £ 15.

121 Zur Art und Weise des Vollzugs dieser Strafen siehe Milner, Nigerian Penal System, S. $111 \mathrm{ff}$.

122 Native Courts Law 1956; siehe dazu Ezejiofor: The Federation of Nigeria. In: Judicial and Legal System in Africa. Hrsg. v. Allott, 2. Aufl. London 1970, S. $77 \mathrm{f}$.

123 States Creation Decree, 1967 - Kano, Kwara, North-Eastern, North-Western, North-Central and Benue Plateau State.

124 G. Ezejiofor: The Federation of Nigeria. In: Judicial and Legal Systems in Afrika. Hrsg. von A. N. Allott, 2. Aufl. London 1972, S. 78 Anm. 19. 
die Richter dieser Gerichte in der neuen Strafprozeßrechtsanwendung ausgebildet wurden, nach dem Criminal Procedure Law von $1960 \mathrm{zu}$ richten.

Die Strafgerichtsbarkeit des customary court in der westlichen Region umfaßt alle Nigerianer; Nicht-Nigerianer unterliegen ihr nicht. Die sachliche Zuständigkeit der Gerichte erstreckt sich nicht auf die schwere Kriminalität; Tötungsdelikte, Hochverrat, Vergewaltigung, Geheimnisverrat, Kuppelei und andere Verbrechen sind ihnen vorenthalten ${ }^{125}$. Die Strafgerichtsbarkeit der customary courts in der Ostregion ist noch begrenzter als in den westlichen Regionen des Landes ${ }^{126}$. Im Staate Lagos gibt es überhaupt keine customary courts mehr.

\section{Schlußbemerkung}

Das Rechts- und Gerichtssystem Nigerias wird zu Recht als kompliziert beschrieben ${ }^{127}$. Der vorliegende Versuch eines Überblicks über Entwicklung und gegenwärtige Lage muß wegen der vielfachen Verästelungen des Systems notwendigerweise lückenhaft und kursorisch bleiben. Der Dualismus im materiellen Strafrecht wird ebenso bedauert und kritisiert wie die fortbestehende Zweispurigkeit des Gerichtwesens im Norden ${ }^{128}$. In bezug auf das materielle Recht wird vorgeschlagen, einen einheitlichen Code für das ganze Land zu schaffen ${ }^{129}$. Ein kleiner Grundstock gesamtnigerianischer Gesetze liegt bereits vor. Es bleibt abzuwarten, ob die neue Militärregierung weiterhin Dekrete für das ganze Land erlassen wird, wie es seit 1966 geschehen ist. Auf diese Weise entstanden zahlreiche gesamtnigerianische Einzelgesetze wie z. B. der Robbery and Fire Arms (Special Provisions) Decree, 1970 und der Indian Hemp Decree, 1966. Ob das offenbar von allen Beteiligten als schwerfällig und nachteilig empfundene dualistische Gerichtssystem ${ }^{130}$ des Nordens abgeschafft wird, läßt sich z. Z. noch nicht übersehen. Ein erster Schritt wurde 1967 mit der Abschaffung der Emir- und Chief-Gerichte getan; auch die Herausnahme der Area Courts aus dem Zuständigkeitsbereich der Eingeborenenverwaltung war vorteilhaft. Die endgültige Aufgabe dieses Rechtszugs ist jedoch noch nicht in Sicht.

\footnotetext{
125 Western Customary Courts Laws 1957, s. 24 (2); zum Rechtszustand im Western State siehe Kayode: Judicial Administration in a Changing Society - Customary Courts in Western Nigeria, in "VRU Bd. 8 (1975), S. 442 f.; zur Zuständigkeit im 1963 gebildeten Mid-Western State siehe Ezejiofor, The Federation of Nigeria, a. a. O., S. 62.

126 Keay and Richardson: The Native and Customary Courts of Nigeria, S. 135.

127 Ezejiofor, The Federation of Nigeria, a. a. O., S. 40.

128 Vgl. Oloyede: The present scope of the Nigerian criminal law. In: Law and Social Change in Nigeria. Hrsg. von T. O. Elias. Lagos 1972, S. 181, 186; Ijaodola: A Case for the Unification of the Court System in Northern Nigeria. In: Nigerian Law Journal 4 (1970), S. 136, 141. Agbede: Towards Unification of Municipal Laws in Africa: Nigerian experience, in „VRU“ Bd. 8 (1975), S. $432 \mathrm{ff}$.

129 Khalil: Criminal Law Reform in Nigeria. In: Journal of Islamic and Comparative Law 5 (1974), S. 51, 54. 130 Zur Kritik siehe Ijaodola: A Case for Unification of Court Systems in Northern Nigeria, S. $141 \mathrm{ff}$.
} 


\title{
Legal Protection of Foreign Investments in Developing Countries
}

\author{
By HeINRICH JüTTNER
}

The demand of the developing countries for full souvereignty over their national ressources und their economic activities takes a great role in the discussion on a new international economic order. Therefore the question of legal protection of foreign investments in the Third World is of great importance too. It is well known indeed, that developing countries as well as industrial countries are interested in foreign investments in developing countries. So they offer a broad catalogue of measures for the legal security of those investments.

The Federal Republic of Germany for example has national guarantees against political risks and bilateral investment protection agreements, until now with 44 developing countries. In the multilateral level there is the "Convention on the Settlement of Investment Disputes between States and Nationals of other States", administered by the World Bank. Against that the "Multilateral Investment Insurance", proposed by the World Bank, and the "Convention on the Protection of Foreign Property", prepared by the OECD, have not yet been realized. The developing countries offer different forms of transfer and property guarantees in their investment laws and arrangements.

Governmental guarantees or insurances against expropriation of foreign investments in developing countries are one of the most important investment incentives. Sometimes the investors are satisfied with the relevant promises of the developing countries; in many cases, however, they effect additional national insurances, because they get a direct claim for compensation in this way. In the Federal Republic of Germany more than $20 \%$ of all foreign investments in the Third World are protected by governmental guarantees against political risks.

The Investment Protection Agreements are classified less important by the investors, because the enforcement of the promises und guarantees in these treaties is depending from the cooperation climate and the legal system in the developing country. Inquiries have come to the result, that there is no incitement effect of these agreements regarding additional foreign investments or investments in certain developing countries. On the other hand their protection effect is positive and generally recognized.

The International Convention for the Settlement of Investment Disputes is regarded as a successful way to strengthen the legal security of foreign investments. So it is already part of many investment treaties, protection agreements and laws. First investment disputes are submit ted to the responsible centre, which has been established by the World Bank. German investors indeed prefere furthermore the governmental German investment guarantees.

\section{Dualism in Criminal Law and Court System in Nigeria}

\section{By BARbara Huber}

There are two criminal codes - with minor modifications in certain states which apply in contemporary Nigeria: The Nigerian Criminal Code of 1916 (NCC) 
applicable in Lagos and the Southern States and The Nigerian Penal Code of 1959 (NPC) which applies in the Northern States. Both codes are derived from the English common law. The different sources of material criminal law is paralleled in the differing approach to criminal procedure and judicial structure.

The purposes of this study would be to trace the historical background which has given rise to modern legal dualism in an African state and at the same time offers a provisional contribution towards the research of basic criminal law principles in Nigeria. The work would be done on the course of a research project, for which the writer is responsible, concerning the treatment of homicide in Northern and Southern Nigeria. The dualism in Nigerian material criminal law is just as much regretted and criticized as the existence of the double tract judical bodies in the Northern States. With reference to the material law, a uniform code for the whole country is proposed. As small foundation for a uniform Nigerian criminal law is already in existence. It remains to be seen whether the new military goverment will henceforth make decrees for the whole country as has been done since 1966 . Wether the dualistic court structure of the North, which is regarded as clumsy and detrimental will be abolished cannot at present be overlooked. The first step in this direction was taken in 1961 when the Emir and Chiefs Courts were abolished. The removal of the Area Courts from the jurisdiction of the Native Authorities was also advantageous. The final abolition of the Area Courts system is however not yet in sight. 